\title{
Fixed-Point Convergence Results of a Three-Step Iterative Process in CAT(0) Spaces
}

\author{
Kifayat Ullah (D), ${ }^{1}$ Junaid Ahmad $\left(D,{ }^{2}\right.$ Muhammad Arshad, ${ }^{2}$ and Manuel De la Sen ${ }^{3}{ }^{3}$ \\ ${ }^{1}$ Department of Mathematics, University of Lakki Marwat, Lakki Marwat 28420, Khyber Pakhtunkhwa, Pakistan \\ ${ }^{2}$ Department of Mathematics and Statistics, International Islamic University, H-10, Islamabad 44000, Pakistan \\ ${ }^{3}$ Institute of Research and Development of Processes, University of the Basque Country, Campus of Leioa (Bizkaia), P.O. Box 644, \\ Bilbao, Barrio Sarriena, Leioa 48940, Spain \\ Correspondence should be addressed to Junaid Ahmad; ahmadjunaid436@gmail.com
}

Received 17 February 2021; Revised 2 March 2021; Accepted 2 June 2021; Published 15 June 2021

Academic Editor: Kamal Shah

Copyright (c) 2021 Kifayat Ullah et al. This is an open access article distributed under the Creative Commons Attribution License, which permits unrestricted use, distribution, and reproduction in any medium, provided the original work is properly cited.

\begin{abstract}
In this article, we suggest some $\Delta$ and strong convergence results of a three-step Sahu-Thakur iteration process for Garcia-Falset maps in the nonlinear setting of CAT(0) spaces. We furnish a new example of Garcia-Falset maps and prove that its three-step Sahu-Thakur iterative process is more effective than the many well-known iterative processes. Our results improve and extend some recently announced results of the current literature.
\end{abstract}

\section{Introduction}

Let $(S, d)$ be a metric space and $\varnothing \neq B \subseteq S$. A self-map $G$ of $B$ is called a contraction map if and only if for every choice of two points $b, b^{\prime} \in B$, there is a real constant $0 \leq r<1$ such that $d\left(G b, G b^{\prime}\right) \leq r d\left(b, b^{\prime}\right)$. The self-map $G$ is regarded as nonexpansive if and only if $d\left(G b, G b^{\prime}\right) \leq d\left(b, b^{\prime}\right)$. A point, namely, $q_{0} \in B$, is called a fixed point for the self-map $G$ of $B$ if and only if $G q_{0}=q_{0}$, and the notation $F_{G}$ will denote the set of all fixed points. In 1922, Banach [1] observed that every self-contraction $G$ of a closed subset $B$ of a Banach space admits a unique fixed point, namely, $q_{0}$, and for every choice of initial value, Picard iterative method (sometimes called the successive approximation method) converges to this $q_{0}$. In 1930, Caccioppoli [2] quickly noted that the Banach result is valid in the setting of general complete metric spaces. The existence of fixed points for a nonexpansive map was independently established by Browder [3] and Göhde [4] in the year 1965. They observed that if $B$ is closed convex and bounded in a uniformly convex Banach space, then $G$ has a fixed point (may not unique). In 2003, Kirk [5] obtained this result in the nonlinear setting of $\mathrm{CAT}(0)$ spaces. Every nonexpansive map is not necessary to be contraction. Hence, the study of fixed points of nonexpansive maps is more harder but more important than the corresponding study of contraction maps. In 2008, Suzuki [6] suggested a very weaker concept of nonexpansive maps. A self-map $G$ of a subset $B$ of a metric space is regarded as a Suzuki map (sometimes called the Suzuki (C)-map) if for any choice of two points $b, b^{\prime} \in B$, one has

$$
\frac{1}{2} d(b, G b) \leq d\left(b, b^{\prime}\right) \Rightarrow d\left(G b, G b^{\prime}\right) \leq d\left(b, b^{\prime}\right) .
$$

Suzuki [6] proved many interesting properties of these maps. He proved that any nonexpansive map is Suzuki, but the inverse is not necessary to be valid, in general. He also generalized the Browder-Gohde result to this new setting of maps. In [7], Nanjaras et al. extended and improved all of the results of Suzuki [6] to the nonlinear setting of CAT(0) spaces. After this, many papers appeared on the topic of Suzuki maps in linear and nonlinear spaces (cf. [8-11] and references therein).

Keeping Suzuki (C)-maps in mind, García-Falset et al. [12] suggested the notion of (E)-maps as follows: a self-map $G$ of a subset $B$ of a metric space is regarded as a Garcia- 
Falset map (sometimes called the Garcia-Falset (E)-map) if for any choice of two points $b, b^{\prime} \in B$, one has

$$
d\left(b, G b^{\prime}\right) \leq \mu d(b, G b)+\left(b, b^{\prime}\right), \quad \text { for some } \mu \geq 1 .
$$

Since by Suzuki [6], every Suzuki map of a nonempty subset $B$ of a metric space satisfies $d\left(b, G b^{\prime}\right) \leq 3 d(b, G b)+$ $\left(b, b^{\prime}\right)$ for every two elements $b, b^{\prime} \in B$. It immediately follows that every Suzuki map is a Garcia-Falset map having real constant $\mu=3$. Interestingly, in this article, we will show that not every Garcia-Falset map is a Suzuki map. Thus, we conclude that the class of Garica-Falset maps properly contains the corresponding class of Suzuki maps. GarcíaFalset et al. [12] established many fixed-point existence results for this larger class of maps.

The existence of fixed points for a self-map when established then to approximate such a fixed point via a most suitable iterative process is not an easy research work. As many know, the unique fixed point of a contraction map can be approximated by using the simplest Picard iterative process [13]. We also know that the fixed point of a nonexpansive map cannot be approximated by using the Picard iterative process, in general. Thus, to obtain a relatively effective rate of convergence and to overcome such type of difficulties, many new iterative processes are designed by authors (see, e.g., Mann [14], Ishikawa [15], Agarwal et al. [16], Noor [17], Abbas and Nazir [18], and Thakur et al. [19]). Among these iterative processes, Sahu et al. [20] and Thakur et al. [21] independently suggested an effective threestep iterative scheme for approximating fixed points of nonexpansive maps:

$$
\left\{\begin{aligned}
b_{1} & =b \in B, \\
g_{k} & =\left(1-\gamma_{k}\right) b_{k}+\gamma_{k} G b_{k}, \\
s_{k} & =\left(1-\beta_{k}\right) g_{k}+\beta_{k} G g_{k}, \\
b_{k+1} & =\left(1-\alpha_{k}\right) G g_{k}+\alpha_{k} G s_{k}, \quad k \in \mathbb{P},
\end{aligned}\right.
$$

where $\alpha_{k}, \beta_{k}, \gamma_{k} \in(0,1)$ and $\mathbb{P}$ denotes the set of all positive integers.

In [21], Thakur et al. established many interesting weak and strong convergence results for the class of nonexpansive maps under iterative process (3) in the ground setting of Banach spaces. Recently in 2019, Usurelu and Postolache [22] improved and extended the main outcome of Thakur et al. [21] to the larger class of Suzuki maps. Very recently in 2020, Usurelu et al. [23] quickly improved and extended the main outcome of Usurelu and Postolache [22] to the general setting of Garcia-Falset maps. The aim of this research work is to improve and extend the main outcome of Usurelu et al. [23] to the nonlinear setting of CAT(0) spaces. Simultaneously, our results improve and extend the corresponding results of Thakur et al. [21] and Usurelu and Postolache [22]. Moreover, we suggest a new example of Garcia-Falset maps which exceeds the corresponding class of Suzuki maps and prove that its Sahu-Thakur iterative process (3) is more effective than the many other iterative schemes.

\section{Preliminaries}

Take a closed interval $[0, v]$ in the set $\mathbb{R}$, and suppose $b, b^{\prime}$ are any elements of a metric space $S=(S, d)$. Then, a map $g:[0, v] \longrightarrow S$ is called geodesic provided that $g(0)=b$, $g(v)=b^{\prime}$, and $d\left(g\left(r_{1}\right), g\left(r_{2}\right)\right)=\left|r_{1}-r_{2}\right|$ for every choice of two points $r_{1}, r_{2} \in[0, v]$. In this case, we regard the image of $g$ as a geodesic segment combining the elements $b$ and $b^{\prime}$, and it is denoted simply by $\left[b, b^{\prime}\right]$ whenever it is unique. Recall that a metric space $S$ is known as a geodesic space if any two points in $S$ are combined by a geodesic. $S$ is called uniquely geodesic in the case when every two elements $b, b^{\prime} \in S$, one can find a unique geodesic combining them. A subset $B$ of $S$ is called convex whenever any two points $b, b^{\prime}$ are given, one is able to combine them by a geodesic in $S$. Notice that the image of each such geodesic always contains in the set $B$.

Now, a given geodesic triangle $\Delta\left(e_{1}, e_{2}, e_{3}\right)$ in a geodesic metric space $S$ is composed of three elements $e_{1}, e_{2}, e_{3}$ in $S$ and a choice of three geodesic segments $\left[e_{1}, e_{2}\right],\left[e_{2}\right.$, $\left.e_{3}\right],\left[e_{3}, e_{1}\right]$ combining them.

A triangle $\bar{\Delta}\left(\bar{e}_{1}, \bar{e}_{2}, \bar{e}_{3}\right)$ in the Euclidean plane $\mathbb{R}^{2}$ is called a comparison triangle for a given geodesic triangle $\Delta\left(e_{1}, e_{2}, e_{3}\right)$ provided that

$$
\begin{aligned}
& d_{\mathbb{R}^{2}}\left(\bar{e}_{1}, \bar{e}_{2}\right)=d\left(e_{2}, e_{3}\right), \\
& d_{\mathbb{R}^{2}}\left(\bar{e}_{2}, \bar{e}_{3}\right)=d\left(e_{2}, e_{3}\right), \\
& d_{\mathbb{R}^{2}}\left(\bar{e}_{3}, \bar{e}_{1}\right)=d\left(e_{3}, e_{1}\right) .
\end{aligned}
$$

An element $\bar{c} \in\left[\bar{e}_{1}, \bar{e}_{2}\right]$ is known as a comparison element for $c \in\left[e_{1}, e_{2}\right]$ provided that $d\left(e_{1}, c\right)=d_{\mathbb{R}^{2}}\left(\bar{e}_{1}, \bar{c}\right)$. Comparison elements on $\left[\bar{e}_{2}, \bar{e}_{3}\right]$ and $\left[\bar{e}_{3}, \bar{e}_{1}\right]$ can be stated in the similar way.

Definition 1. Suppose $\Delta\left(e_{1}, e_{2}, e_{3}\right)$ is a given geodesic triangle in a metric space $(S, d)$. We say that $\Delta\left(e_{1}, e_{2}, e_{3}\right)$ is endowed with the $\mathrm{CAT}(0)$ inequality whenever for $e, e^{\prime} \in \Delta\left(e_{1}, e_{2}, e_{3}\right)$ and for their comparison elements, namely, $\bar{e}, e^{\prime} \in \bar{\Delta}\left(\bar{e}_{1}, \bar{e}_{2}, \bar{e}_{3}\right)$, it follows that

$$
d(e, e l)=d_{\mathbb{R}^{2}}\left(\bar{e}, \bar{e}^{\prime}\right) .
$$

Notice that a geodesic space $S$ is called a CAT(0) space provided that each of its geodesic triangle is endowed with the $\mathrm{CAT}(0)$ inequality. Some other equivalent definition of CAT(0) can be found in [24]. Moreover, each CAT(0) space is uniquely geodesic. Some well-known examples of CAT(0) spaces include pre-Hilbert spaces, metric trees, and Euclidean buildings. For more details and the literature of fixed-point theory in nonlinear spaces, we refer the reader to [24-27].

We now state a result from [28].

Lemma 1. Suppose $(S, d)$ is a given $C A T(0)$ space.

(i) If $b, b^{\prime} \in S$ and $\kappa \in[0,1]$ is fixed, then there exists a unique element, namely, $g \in\left[b, b^{\prime}\right]$, such that 


$$
\begin{aligned}
d(b, g) & =\kappa d\left(b, b^{\prime}\right), \\
d\left(b^{\prime}, g\right) & =(1-\kappa) d\left(b, b^{\prime}\right) .
\end{aligned}
$$

We shall normally represent by $(1-\kappa) b \oplus \kappa b^{\prime}$ the unique element $g$ satisfying (6).

(ii) If $b, b^{\prime}, a \in S$ and $\kappa \in[0,1]$ is fixed, then

$$
d\left(a, \kappa b \oplus(1-\kappa) b^{\prime}\right) \leq \kappa d(a, b)+(1-\kappa) b^{\prime} .
$$

Assume that $\varnothing \neq B \subseteq S$ is convex and closed in a CAT(0) space and $\left\{b_{k}\right\} \subseteq S$ is bounded. Fix $b \in S$, and set

$$
r\left(b,\left\{b_{k}\right\}\right)=\limsup _{k \rightarrow \infty} d\left(b_{k}, b\right) .
$$

We normally denote and define the asymptotic radius of the sequence $\left\{b_{k}\right\}$ wrt $B$ as

$$
r\left(B,\left\{b_{k}\right\}\right)=\inf \left\{r\left(b,\left\{b_{k}\right\}\right): b \in B\right\} .
$$

We normally denote and define the asymptotic center of the sequence $\left\{b_{k}\right\}$ wrt $B$ as

$A\left(B,\left\{b_{k}\right\}\right)=\left\{b \in B: r\left(b,\left\{b_{k}\right\}\right)=r\left(B,\left\{b_{k}\right\}\right)\right\}$.

It is known that the asymptotic center is simply denoted by $A\left(\left\{b_{k}\right\}\right)$ wrt $S$. Interestingly, the set $A\left(B,\left\{b_{k}\right\}\right)$ contains unique elements in the setting of complete CAT(0) spaces (see [29]).

Definition 2. A bounded sequence $\left\{b_{k}\right\}$ of elements of a CAT( 0$)$ space $S$ is called $\Delta$-convergent to $q_{0} \in S$ provided that $q_{0}$ is the unique asymptotic center for every subsequence $\left\{s_{k}\right\}$ of $\left\{b_{k}\right\}$. When $q_{0}$ is $\Delta-\lim$ of $\left\{b_{k}\right\}$, then we write $\Delta-\lim _{k} b_{k}=q_{0}$.

Every CAT(0) space $S$ enjoys the Opial-like property [30], that is, if $\left\{b_{k}\right\} \subseteq S$ is $\Delta$-convergent to $q_{0}$, then for every choice of $p_{0} \neq q_{0} \in S$, it follows that

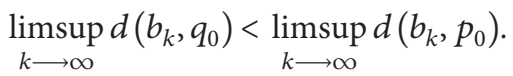

Lemma 2 (see [31]). Suppose $S$ is a CAT(0) space and $\left\{b_{k}\right\} \subseteq S$ is bounded. Then, $\left\{b_{k}\right\}$ always has a $\Delta$-convergent subsequence.

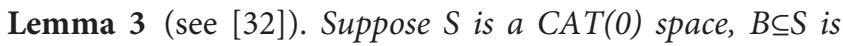
closed and convex, and $\left\{b_{k}\right\} \subseteq B$ is bounded. Then, the asymptotic center of the sequence $\left\{b_{k}\right\}$ is always in $B$.

The following characterizations of Garcia-Falset maps can be found in [12].

Proposition 1. Let $B$ be a nonempty subset of a complete $C A T(0)$ space and $G: B \longrightarrow B$. (i) If $G$ is a Suzuki mapping, then $G$ is also a GarciaFalset map

(ii) If $G$ is a Garcia-Falset map having $F_{G} \neq \varnothing$, then for every choice of $b \in B$ and $q_{0} \in F_{G}$, one has $d\left(G b, G q_{0}\right) \leq d\left(b, q_{0}\right)$

(iii) If $G$ is a Garcia-Falset map, then the set $F_{G}$ is closed

We know the following characterization of the CAT(0) space from [33].

Lemma 4. Assume that $(S, d)$ is a given complete $C A T(0)$ space, and fix $b \in S$. Suppose $0<y \leq \eta_{k} \leq z<1$ and $\left\{r_{k}\right\},\left\{s_{k}\right\}$ are two sequences of elements of $S$ such that $\limsup _{k \rightarrow \infty} d\left(r_{k}, b\right) \leq \xi, \quad \limsup _{k \rightarrow \infty} d\left(s_{k}, b\right) \leq \xi, \quad$ and $\lim _{k \rightarrow \infty} d\left(\eta_{k} r_{k} \oplus\left(1-\eta_{k}\right) s_{k}, b\right)=\xi$ for some real number $\xi \geq 0$; then, $\lim _{k \longrightarrow \infty} d\left(r_{k}, s_{k}\right)=0$.

\section{Convergence Theorems in CAT(0) Spaces}

The aim of this section is to establish $\Delta$ and strong convergence of the Sahu-Thakur iterative scheme for GarciaFalset maps. Throughout the section, we shall denote simply by $S$ a complete CAT(0) space. Notice that Sahu-Thakur iterative process (3) in the framework of CAT(0) spaces is written as follows:

$$
\left\{\begin{aligned}
b_{1} & =b \in B, \\
g_{k} & =\left(1-\gamma_{k}\right) b_{k} \oplus \gamma_{k} G b_{k}, \\
s_{k} & =\left(1-\beta_{k}\right) g_{k} \oplus \beta_{k} G g_{k}, \\
b_{k+1} & =\left(1-\alpha_{k}\right) G g_{k} \oplus \alpha_{k} G s_{k}, \quad k \in \mathbb{P},
\end{aligned}\right.
$$

where $\alpha_{k}, \beta_{k}, \gamma_{k} \in(0,1)$.

We first establish a key lemma as follows.

Lemma 5. Suppose B be any nonempty closed convex subset of $S$, and assume that $G: B \longrightarrow B$ is a Garcia-Falset map having $F_{G} \neq \varnothing$. If the sequence $\left\{b_{k}\right\}$ is defined by equation (12), then $\lim _{k \longrightarrow \infty} d\left(b_{k}, q_{0}\right)$ exists for each choice of $q_{0} \in F_{G}$.

Proof. Suppose $q_{0} \in F_{G}$. Keeping Proposition 1 (ii) in mind, one has

$$
\begin{aligned}
d\left(g_{k}, q_{0}\right) & =d\left(\left(1-\gamma_{k}\right) b_{k} \oplus \gamma_{k} G b_{k}, q_{0}\right) \\
& \leq\left(1-\gamma_{k}\right) d\left(b_{k}, q_{0}\right)+\gamma_{k} d\left(G b_{k}, q_{0}\right) \\
& \leq\left(1-\gamma_{k}\right) d\left(b_{k}, q_{0}\right)+\gamma_{k} d\left(b_{k}, q_{0}\right) \\
& \leq d\left(b_{k}, q_{0}\right), \\
d\left(s_{k}, q_{0}\right) & =d\left(\left(1-\beta_{k}\right) g_{k} \oplus \beta_{k} G g_{k}, q_{0}\right) \\
& \leq\left(1-\beta_{k}\right) d\left(g_{k}, q_{0}\right)+\beta_{k} d\left(G g_{k}, q_{0}\right) \\
& \leq\left(1-\beta_{k}\right) d\left(g_{k}, q_{0}\right)+\beta_{k} d\left(g_{k}, q_{0}\right) \\
& \leq d\left(g_{k}, q_{0}\right) .
\end{aligned}
$$

Using the above inequalities, we have 


$$
\begin{aligned}
d\left(b_{k+1}, q_{0}\right) & =d\left(\left(1-a_{k}\right) G g_{k} \oplus \alpha_{k} G s_{k}, q_{0}\right) \\
& \leq\left(1-\alpha_{k}\right) d\left(G g_{k}, q_{0}\right)+\alpha_{k} d\left(G s_{k}, q_{0}\right) \\
& \leq\left(1-\alpha_{k}\right) d\left(g_{k}, q_{0}\right)+\alpha_{k} d\left(s_{k}, q_{0}\right) \\
& \leq\left(1-\alpha_{k}\right) d\left(g_{k}, q_{0}\right)+\alpha_{k} d\left(g_{k}, q_{0}\right) \\
& =d\left(g_{k}, q_{0}\right) \leq d\left(b_{k}, q_{0}\right) .
\end{aligned}
$$

Hence, the real sequence $\left\{d\left(b_{k}, q_{0}\right)\right\}$ is nonincreasing and bounded. Thus, we conclude that $\lim _{k \rightarrow \infty} d\left(b_{k}, q_{0}\right)$ exists for each choice of $q_{0} \in F_{G}$.

Using Lemma 5 and the definition of the Garcia-Falset map, we establish the following result in $\mathrm{CAT}(0)$ spaces.

Theorem 1. Let $B$ be a nonempty convex closed subset of $S$, and let $G: B \longrightarrow B$ be a Garcia-Falset operator. For arbitrary chosen $b_{1} \in B$, let the sequence $\left\{b_{k}\right\}$ be defined by equation (12). Then, $F_{G} \neq \varnothing$ if and only if $\left\{b_{k}\right\}$ is bounded and $\lim _{k \longrightarrow \infty} d\left(b_{k}, G b_{k}\right)=0$.

Proof. Suppose that the sequence $\left\{b_{k}\right\}$ is bounded and $\lim _{k \rightarrow \infty} d\left(b_{k}, G b_{k}\right)=0$. Fix an element $q_{0} \in A\left(B,\left\{b_{k}\right\}\right)$. We are going to show that $q_{0}=G q_{0}$. Since $G$ is a Garcia-Falset map,

$$
\begin{aligned}
r\left(G q_{0},\left\{b_{k}\right\}\right) & =\limsup _{k \longrightarrow \infty} d\left(b_{k}, G q_{0}\right) \\
& \leq \mu \limsup _{k \rightarrow \infty} d\left(b_{k}, G b_{k}\right)+\limsup _{k \longrightarrow \infty} d\left(b_{k}, q_{0}\right) \\
& =\limsup _{k \longrightarrow \infty} d\left(b_{k}, q_{0}\right)=r\left(q_{0},\left\{b_{k}\right\}\right) .
\end{aligned}
$$

Hence, it is seen that $G q_{0} \in A\left(B,\left\{b_{k}\right\}\right)$, but $A\left(B,\left\{b_{k}\right\}\right)$ is singleton, and one can conclude that $q_{0}=G q_{0}$. Thus, $q_{0}$ is the element of $F_{G}$.

Conversely, let the set $F_{G} \neq \varnothing$. We are going to show that $\left\{b_{k}\right\}$ is bounded and $\lim _{k \rightarrow \infty} d\left(b_{k}, G b_{k}\right)=0$. Fix $q_{0} \in F_{G}$; then, by Lemma $5, \lim _{k \rightarrow \infty} d\left(b_{k}, q_{0}\right)$ exists, and $\left\{b_{k}\right\}$ is bounded. Put

$$
\lim _{k \longrightarrow \infty} d\left(b_{k}, q_{0}\right)=\xi
$$

The proof of Lemma 5 suggests that

$$
\begin{array}{r}
d\left(g_{k}, q_{0}\right) \leq d\left(b_{k}, q_{0}\right) \\
\Rightarrow \limsup _{k \rightarrow \infty} d\left(g_{k}, q_{0}\right) \leq \limsup _{k \rightarrow \infty} d\left(b_{k}, q_{0}\right)=\xi .
\end{array}
$$

By using Proposition 1 (ii), one has

$$
\limsup _{k \rightarrow \infty} d\left(G b_{k}, q_{0}\right) \leq \limsup _{k \rightarrow \infty} d\left(b_{k}, q_{0}\right)=\xi .
$$

Also, the proof of Lemma 5 suggests that

$$
\begin{aligned}
& d\left(b_{k+1}, q_{0}\right) \leq d\left(g_{k}, q_{0}\right) \\
& \Rightarrow \xi \leq \liminf _{k \longrightarrow \infty} d\left(g_{k}, q_{0}\right) .
\end{aligned}
$$

From equations (17) and (19), we obtain

$$
\xi=\lim _{k \longrightarrow \infty} d\left(g_{k}, q_{0}\right) .
$$

From equation (20), we have

$$
\xi=\lim _{k \longrightarrow \infty} d\left(g_{k}, q_{0}\right)=\lim _{k \longrightarrow \infty} d\left(\left(1-\gamma_{k}\right) b_{k} \oplus \gamma_{k} G b_{k}, q_{0}\right) .
$$

Applying Lemma 4, we obtain

$$
\lim _{k \longrightarrow \infty} d\left(b_{k}, G b_{k}\right)=0 .
$$

This completes the proof.

We now establish a strong convergence for Garcia-Falset mappings on compact domains in $\mathrm{CAT}(0)$ spaces.

Theorem 2. Assume that B is a nonempty convex compact subset of S. Suppose $G$ and $\left\{b_{k}\right\}$ are as given in Theorem 1 and $F_{G} \neq \varnothing$. Then, $\left\{b_{k}\right\}$ converges strongly to a point of $F_{G}$.

Proof. By the compactness property of the set $B$, we can construct a subsequence $\left\{b_{k_{1}}\right\}$ of $\left\{b_{k}\right\}$ such that $\lim _{l \rightarrow \infty} d\left(b_{k_{k}}, p_{0}\right)=0$, for some $p_{0} \in B$. Since the self-map $G$ is a Garcia-Falset map, there exists a real constant $\mu \geq 1$, with

$$
d\left(b_{k_{l}}, G p_{0}\right) \leq \mu d\left(b_{k_{l}}, G b_{k_{l}}\right)+d\left(b_{k_{l}}, p_{0}\right) \text {. }
$$

In the view of Theorem $1, \lim _{l \rightarrow \infty} d\left(b_{k_{l}}, G b_{k_{l}}\right)=0$. Now, using $\lim _{l \longrightarrow \infty} d\left(b_{k_{l}}, G b_{k_{l}}\right)=0$ and $\lim _{l \rightarrow \infty} d\left(b_{k_{l}}, p_{0}\right)=0$, we have from equation (23) $\lim _{l \rightarrow \infty} d\left(b_{k_{l}}, G p_{0}\right)=0$, but $S$ is a metric space, so the limit of $\left\{b_{k_{l}}\right\}$ is unique, that is, $G p_{0}=p_{0}$. Hence, $p_{0} \in F_{G}$. By Lemma 5, $\lim _{k \longrightarrow \infty} d\left(b_{k}, p_{0}\right)$ exists. Hence, $p_{0}$ is the strong limit of $\left\{b_{k}\right\}$.

Theorem 3. Assume that $B$ is a nonempty closed convex subset of S. Suppose $G$ and $\left\{b_{k}\right\}$ are as given in Theorem 1 . If $F_{G} \neq \varnothing$ and $\liminf _{k \rightarrow \infty} d\left(b_{k}, F_{G}\right)=0$, then $\left\{b_{k}\right\}$ converges strongly to a point of $F_{G}$.

Proof. Since the proof is elementary, we skip it.

We now give the complete definition of condition $I$, which was originally introduced by Sentor and Dotson in [34].

Definition 3. Assume that $B$ is a nonempty subset of $S$. One says that a self-map $G$ of $B$ has condition $I$ whenever there is a nondecreasing map $\mu$ which satisfies $\mu(p)=0$ if and only if $p=0$ and $\mu(p)>0$ for every choice of $p>0$ and $d(b, G b) \geq \mu\left(d\left(b, F_{G}\right)\right)$ for each choice of $b \in B$.

Now, we state and prove a strong convergence result based on condition $I$.

Theorem 4. Assume that $B$ is a nonempty closed convex subset of S. Suppose $G$ and $\left\{b_{k}\right\}$ are as given in Theorem 1 and $F_{G} \neq \varnothing$. Then, $\left\{b_{k}\right\}$ converges strongly to a point of $F_{G}$ if $G$ has condition I.

Proof. The conclusions of Theorem 1 provide us 


$$
\liminf _{k \longrightarrow \infty} d\left(b_{k}, G b_{k}\right)=0 .
$$

Condition $I$ of $G$ suggests

$$
d\left(b_{k}, G b_{k}\right) \geq \mu\left(d\left(b_{k}, F_{G}\right)\right) \text {. }
$$

Using equation (24) and condition $I$, one has

$$
\liminf _{k \longrightarrow \infty} \mu\left(d\left(b_{k}, F_{G}\right)=0\right) .
$$

However, the map $\mu:[0, \infty) \longrightarrow[0, \infty)$ is nondecreasing with $\mu(0)=0$ and $\mu(p)>0$ for each choice of $p>0$, so

$$
\liminf _{k \longrightarrow \infty} d\left(b_{k}, F_{G}\right)=0 .
$$

The conclusions of Theorem 3 suggest that $\left\{b_{k}\right\}$ is strongly convergent in the set $F_{G}$.

Finally, we suggest $\Delta$-convergence for maps having (E)condition.

Theorem 5. Assume that $B$ is a nonempty closed convex subset of S. Suppose $G$ and $\left\{b_{k}\right\}$ are as given in Theorem 1 and $F_{G} \neq \varnothing$. Then, $\left\{b_{k}\right\} \Delta$ converges to a point of $F_{G}$.

Proof. By Theorem 1, one can conclude that $\left\{b_{k}\right\}$ is bounded and is such that $\lim _{k \rightarrow \infty} d\left(b_{k}, G b_{k}\right)=0$. We may suppose that $\omega_{\Delta}\left(\left\{b_{k}\right\}\right)=\bigcup A\left(\left\{s_{k}\right\}\right)$, for every subsequence $\left\{s_{k}\right\}$ of $\left\{b_{k}\right\}$. The next purpose is to obtain $\omega_{\Delta}\left(\left\{b_{k}\right\}\right) \subseteq F_{G}$. If we select any $s \in \omega_{\Delta}\left(\left\{b_{k}\right\}\right)$, then there exists a subsequence, namely, $\left\{s_{k}\right\}$, of $\left\{b_{k}\right\}$ with the property $A\left(\left\{s_{k}\right\}\right)=\{s\}$. Using Lemmas 2 and 3 , we may conclude that a subsequence $\left\{r_{k}\right\}$ of $\left\{s_{k}\right\}$ exists having some $\Delta$-limit $r$ in $B$, but Theorem 1 suggests that $\lim _{k \rightarrow \infty} d\left(r_{k}, G r_{k}\right)=0$, and also, $G$ is endowed with the (E)-condition, so we have

$$
d\left(r_{k}, G r\right) \leq \mu d\left(r_{k}, G r_{k}\right)+d\left(r_{k}, r\right) .
$$

By considering lim sup on both sides of equation (28), we obtain $r \in F_{G}$. Lemma 5 suggests that $\lim _{k \rightarrow \infty} d\left(r_{k}, r\right)$ exists. We need to show that $s=r$. Assume, on the contrary, that $s$ is different from $r$. So, by uniqueness of asymptotic centers, one has

$$
\begin{aligned}
& \limsup _{k \rightarrow \infty} d\left(r_{k}, r\right)<\limsup _{k \longrightarrow \infty} d\left(r_{k}, s\right) \leq \limsup _{k \longrightarrow \infty} d\left(s_{k}, s\right) \\
&<\limsup _{k \longrightarrow \infty} d\left(s_{k}, r\right)=\limsup _{k \longrightarrow \infty} d\left(b_{k} \cdot r\right) \\
&=\limsup _{k \longrightarrow \infty} d\left(r_{k}, r\right) .
\end{aligned}
$$

Consequently,

$\limsup _{k \rightarrow \infty} d\left(r_{k}, r\right)<\limsup _{k \rightarrow \infty} d\left(r_{k}, r\right)$. This is a contradiction, and so, one can conclude that $s=r \in F_{G}$ and $\omega_{\Delta}\left(\left\{b_{k}\right\}\right) \subseteq F_{G}$.
It is now remaining to prove $\left\{b_{k}\right\}$ is a $\Delta$-convergent sequence in the set $F_{G}$. For this purpose, we need $\omega_{\Delta}\left(\left\{b_{k}\right\}\right)$ which is a singleton set. If $\left\{s_{k}\right\}$ is any subsequence of $\left\{b_{k}\right\}$, then by Lemmas 2 and 3, we can choose the $\Delta$-convergent subsequence $\left\{r_{k}\right\}$ of $\left\{s_{k}\right\}$ having some $\Delta-\lim r$ in $B$. Assume that $A\left(\left\{s_{k}\right\}\right)=\{s\}$ and $A\left(\left\{b_{k}\right\}\right)=\{q\}$. We have already showed that $s=r$ and $r \in F_{G}$. We are going to prove that $q=r$. Suppose not, then $\lim _{k \rightarrow \infty} d\left(b_{k}, r\right)$ exists, and also, the asymptotic centers are singleton, and we have

$$
\begin{aligned}
& \limsup _{k \longrightarrow \infty} d\left(r_{k}, r\right)<\underset{k \longrightarrow \infty}{\limsup _{k}} d\left(r_{k}, q\right) \leq \limsup _{k \longrightarrow \infty} d\left(b_{k}, q\right) \\
& <\limsup _{k \rightarrow \infty} d\left(b_{k}, r\right)=\underset{k \longrightarrow \infty}{\limsup } d\left(r_{k} \cdot r\right) \text {, }
\end{aligned}
$$

which is a contradiction. Thus, $q=r \in F_{G}$. Hence, $\omega_{\Delta}(\{b\})=\{q\}$. This completes the proof.

\section{Numerical Example}

We now furnish a new numerical example of Garcia-Falset maps and show that its Sahu-Thakur iterative scheme is more effective than the other well-known existing iterative schemes.

Example 1. We may set a self-map $G$ of $B=[4,10]$ by the rule $G b=((b+4) / 2)$ for $b \in B_{1}=[4,10)$ and $G b=4$ for $b \in B_{2}=\{10\}$. To observe that the map $G$ is a Garcia-Falset map, we will establish $d\left(b, G b^{\prime}\right) \leq \mu d(b, G b)+d\left(b, b^{\prime}\right)$ for every choice of $b, b^{\prime} \in B$ and some $\mu \geq 1$. We choose $\mu=2$ and divide the proof in parts as follows:

$$
\begin{aligned}
& \left(P_{1}\right) \text { Let } b, b^{\prime} \\
& \begin{aligned}
d\left(b, G b^{\prime}\right) & =\left|b-G b^{\prime}\right|=|b-4|=|b-G b| \\
& \leq 2|b-G b| \leq 2|b-G b|+\left|b-b^{\prime}\right| \\
& =2 d(b, G b)+d\left(b, b^{\prime}\right)
\end{aligned}
\end{aligned}
$$

$\left(P_{2}\right) \quad$ Let $\quad b, b^{\prime} \in B_{1}$, so $\quad G b=((b+4) / 2) \quad$ and $G b^{\prime}=\left(\left(b^{\prime}+4\right) / 2\right)$. Then,

$$
\begin{aligned}
d\left(b, G b^{\prime}\right) & =\left|b-G b^{\prime}\right| \leq|b-G b|+\left|G b-G b^{\prime}\right| \\
& =|b-G b|+\left|\left(\frac{b+4}{2}\right)-\left(\frac{b^{\prime}+4}{2}\right)\right| \\
& =|b-G b|+\frac{1}{2}\left|b-b^{\prime}\right| \\
& \leq|b-G b|+\left|b-b^{\prime}\right| \\
& \leq 2|b-G b|+\left|b-b^{\prime}\right| .
\end{aligned}
$$


TABle 1: Some values generated by Picard, Mann, Ishikawa, Noor, Agarwal, Abbas, Thakur, and Sahu-Thakur (ST) iterative schemes to $q_{0}=4$ of the mapping $G$ as given in Example 1 .

\begin{tabular}{lcccccccc}
\hline$k$ & Picard & Mann & Ishikawa & Noor & Agarwal & Abbas & Thakur & ST \\
\hline 1 & 4.5 & 4.5 & 4.5 & 4.5 & 4.5 & 4.5 & 4.5 & 4.5 \\
2 & 4.2500 & 4.2875 & 4.2184 & 4.2029 & 4.1809 & 4.1807 & 4.1487 \\
3 & 4.1250 & 4.1653 & 4.0954 & 4.0823 & 4.0655 & 4.0653 & 4.0442 \\
4 & 4.0625 & 4.0950 & 4.0416 & 4.0334 & 4.0236 & 4.0236 & 4.0131 & 4.0393 \\
5 & 4.0313 & 4.0546 & 4.0182 & 4.0136 & 4.0085 & 4.0085 & 4.0039 & 4.0110 \\
6 & 4.0156 & 4.0314 & 4.0079 & 4.0055 & 4.0031 & 4.0031 & 4.0012 \\
7 & 4.0078 & 4.0180 & 4.0034 & 4.0022 & 4.0011 & 4.0011 & 4.0003 \\
8 & 4.0039 & 4.0103 & 4.0015 & 4.0009 & 4.0004 & 4.0004 & 4.0001 \\
9 & 4.0020 & 4.0059 & 4.0006 & 4.0003 & 4.0001 & 4.0001 & 4.0000 \\
10 & 4.0010 & 4.0034 & 4.0002 & 4.0001 & 4.0000 & 4.0000 & 4.0000 \\
11 & 4.0005 & 4.0019 & 4.0001 & 4.0000 & 4.0000 & 4.0000 & 4.00000 \\
12 & 4.0002 & 4.0011 & 4.0000 & 4.0000 & 4.0000 & 4.0000 & 4.00000 \\
13 & 4.0001 & 4.0006 & 4.0000 & 4.0000 & 4.0000 & 4.0000 & 4.0000 \\
14 & 4.0000 & 4.0004 & 4.0000 & 4.0000 & 4.0000 & 4.0000 & 4.000000 \\
15 & 4.0000 & 4.0002 & 4.0000 & 4.0000 & 4.0000 & 4.0000 & 4.000000 \\
16 & 4.0000 & 4.0001 & 4.0000 & 4.0000 & 4.0000 & 4.0000 & 4.0000 \\
17 & 4.0000 & 4.0000 & 4.0000 & 4.0000 & 4.0000 & 4.0000 & 4.0000 \\
\hline
\end{tabular}

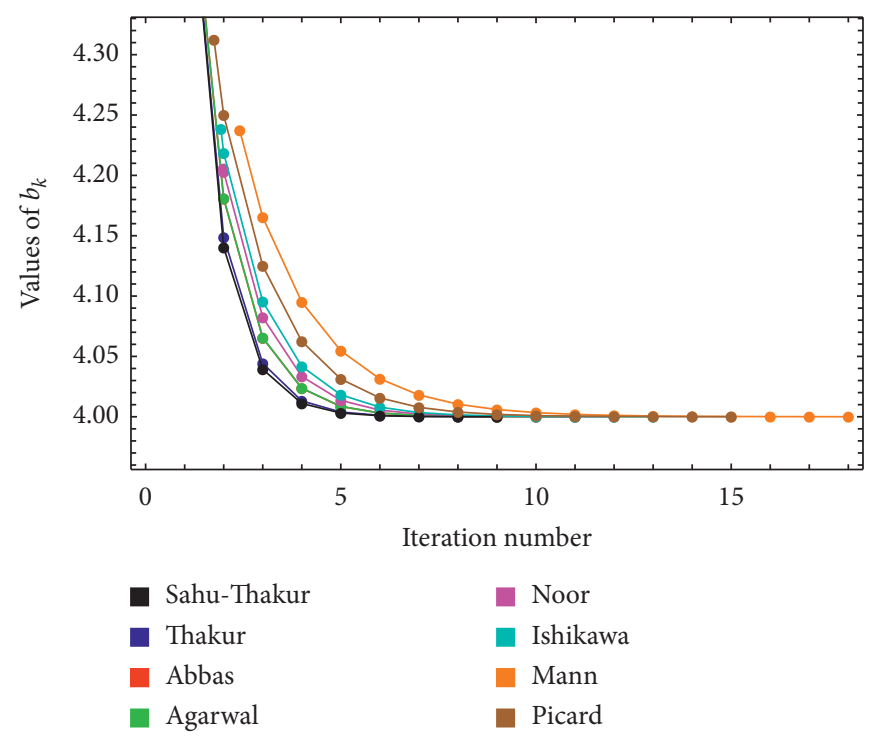

FIGURE 1: Convergence behaviors of different iterative schemes to a fixed point $q_{0}=4$ of the self-map $G$.

$\left(P_{3}\right)$ Let $b \in B_{1}$ and $b^{\prime} \in B_{2}$, so $G b=((b+4) / 2)$ and $G b^{\prime}=4$. Then,

$$
\begin{aligned}
d\left(b, G b^{\prime}\right) & =\left|b-G b^{\prime}\right|=|b-4|=2\left|\frac{b-4}{2}\right| \\
& =2\left|b-\left(\frac{b+4}{2}\right)\right| \\
& =2|b-G b| \leq 2|b-G b|+\left|b-b^{\prime}\right| \\
& =2 d(b, G b)+d\left(b, b^{\prime}\right) .
\end{aligned}
$$

$\left(P_{4}\right)$ Let $b \in B_{2}$ and $b^{\prime} \in B_{1}$, so $G b^{\prime}=\left(\left(b^{\prime}+4\right) / 2\right)$ and $G b=4$. Then,

$$
\begin{aligned}
d\left(b, G b^{\prime}\right) & =\left|b-G b^{\prime}\right|=\left|b-\left(\frac{b^{\prime}+4}{2}\right)\right| \\
& =\left|\frac{b+b-b^{\prime}-4}{2}\right| \leq\left|\frac{b-4}{2}\right|+\left|\frac{b-b^{\prime}}{2}\right| \\
& =\frac{1}{2}|b-G b|+\frac{1}{2}\left|b-b^{\prime}\right| \leq 2|b-G b|+\left|b-b^{\prime}\right| \\
& =2 d(b, G b)+d\left(b, b^{\prime}\right) .
\end{aligned}
$$

The cases discussed above show that $G$ is a Garcia-Falset map on the set $B$. By choosing $b=8.2$ and $b^{\prime}=10$, we may 
observe that $(1 / 2) d(b, G b) \leq d\left(b, b^{\prime}\right) \quad$ and $d\left(G b, G b^{\prime}\right)>d\left(b, b^{\prime}\right)$, that is, $G$ is not a Suzuki map on $B$. We now choose $\alpha_{k}=0.85$ and $\beta_{k}=0.65$ and $\gamma_{k}=0.45$ for every natural number $k$. In Table 1 , strong convergence of Sahu-Thakur iteration (ST) $[20,21]$ is compared with the other existing iterative schemes such as Picard [35], Mann [14], Ishikawa [15], Noor [17], Agarwal et al. et al. [16], Abbas and Nazir [18], and Thakur et al. [19]. Both Table 1 and Figure 1 well suggest the effectiveness of Sahu-Thakur iteration in the class of Garcia-Falset maps.

\section{Conclusion}

The extension of fixed-point results from the context of the linear domain to the general context of the nonlinear domain has its own significance. In [36], Takahashi suggested the notion of convexity in metric spaces and studied some fixed-point results in this context. After this, many different convexity structures in a context of metric spaces are introduced. In [23], Usurelu et al. established some convergence results for Garcia-Falset maps under Sahu-Thakur in the linear context of Banach spaces. In this paper, we have extended their results to the nonlinear context of CAT(0) spaces. We have also suggested a new example of GarciaFalset and showed that its Sahu-Thakur iterative process is more effective than the other prominent iterative schemes. Keeping the previous discussion in mind, the cases when the map is Suzuki or else is nonexpansive are now special cases of our main results. Thus, our results simultaneously improve and extend the corresponding results in [7-9, 11, 18-22, 37] from the setting of nonexpansive and Suzuki maps to the setting of Garcia-Falset maps and from the linear domain to the general setting of the nonlinear domain [38].

\section{Data Availability}

No data were used to support this study.

\section{Conflicts of Interest}

The authors declare that they have no conflicts of interest.

\section{Authors' Contributions}

All the authors contributed equally to this article.

\section{Acknowledgments}

The authors are grateful to the Basque Government for its support through grant IT1207-19.

\section{References}

[1] S. Banach, "Sur les opérations dans les ensembles abstraits et leur application aux équations intégrales," Fundamenta Mathematicae, vol. 3, pp. 133-181, 1922.

[2] R. Caccioppoli, "Un teorema generale sull esistenza di elementi uniti in unatransformazione funzionale," Rendiconti Lincei Mathematica e Applicazioni, vol. 11, pp. 794-799, 1930.
[3] F. E. Browder, "Nonexpansive nonlinear operators in a banach space," Proceedings of the National Academy of Sciences, vol. 54, no. 4, pp. 1041-1044, 1965.

[4] D. Göhde, "Zum prinzip der kontraktiven abbildung," Mathematische Nachrichten, vol. 30, no. 3-4, pp. 251-258, 1965.

[5] W. A. Kirk, "Geodesic geometry and fixed point theory in: seminar of mathematical analysis (Malaga/Seville, 2002/ 2003), colecc. Abierta, univ. Sevilla secr. Publ," Seville, vol. 64, pp. 195-225, 2003.

[6] T. Suzuki, "Fixed point theorems and convergence theorems for some generalized nonexpansive mappings," Journal of Mathematical Analysis and Applications, vol. 340, no. 2, pp. 1088-1095, 2008.

[7] B. Nanjaras, B. Panyanak, and W. Phuengrattana, "Fixed point theorems and convergence theorems for suzuki-generalized nonexpansive mappings in CAT (0) spaces," Nonlinear Analysis Hybrid Systems, vol. 4, pp. 25-31, 2020.

[8] J. Ali, F. Ali, and P. Kumar, "Approximation of fixed points for suzuki's generalized non-expansive mappings," Mathematics, vol. 7, no. 6, p. 522, 2019.

[9] M. Basarir and A. Sahin, "On the strong and $\Delta$-convergence of S-iteration process for generalized nonexpansive mappings on CAT (0) spaces," Thai Journal of Mathematics, vol. 12, pp. 549-559, 2014.

[10] S. Hassan, M. de la Sen, P. Agarwal, Q. Ali, and A. Hussain, “A new faster iterative scheme for numerical fixed points estimation of suzukis generalized nonexpansive mappings," Mathematical Problems in Engineering, vol. 2020, Article ID 38638, 9 pages, 2020.

[11] W. Phuengrattana, "Approximating fixed points of suzukigeneralized nonexpansive mappings," Nonlinear Analysis: Hybrid Systems, vol. 5, no. 3, pp. 583-590, 2011.

[12] J. García-Falset, E. Llorens-Fuster, and T. Suzuki, "Fixed point theory for a class of generalized nonexpansive mappings," Journal of Mathematical Analysis and Applications, vol. 375, no. 1, pp. 185-195, 2011.

[13] P. Agarwal, M. Jleli, and B. Samet, Fixed Point Theory in Metric Spaces, Springer Nature Singapore Private Limited, Singapore, 2018.

[14] W. R. Mann, "Mean value methods in iteration," Proceedings of the American Mathematical Society, vol. 4, no. 3, p. 506, 1953.

[15] S. Ishikawa, "Fixed points by a new iteration method," Proceedings of the American Mathematical Society, vol. 44, no. 1, pp. 147-150, 1974.

[16] R. P. Agarwal, D. O’Regon, and D. R. Sahu, "Iterative construction of fixed points of nearly asymtotically nonexpansive mappings," Journal of Nonlinear and Convex Analsis, vol. 8, pp. 61-79, 2007.

[17] M. A. Noor, "New approximation schemes for general variational inequalities," Journal of Mathematical Analysis and Applications, vol. 251, no. 1, pp. 217-229, 2000.

[18] M. Abbas and T. Nazir, "A new faster iteration process applied to constrained minimization and feasibility problems," Matematički Vesnik, vol. 66, pp. 223-234, 2014.

[19] B. S. Thakur, D. Thakur, and M. Postolache, "New iteration scheme for numerical reckoning fixed points of nonexpansive mappings," Journal of Inequalities and Applications, vol. 328, 2014.

[20] V. K. Sahu, H. K. Pathak, and R. Tiwari, "Convergence theorems for new iterations cheme and comparison results," Aligarh Bulletin of Mathematics, vol. 35, pp. 19-42, 2016. 
[21] B. Thakur, D. Thakur, and M. Postolache, "A new iteration scheme for approximating fixed points of nonexpansive mappings," Filomat, vol. 30, no. 10, pp. 2711-2720, 2016.

[22] G. I. Usurelu and M. Postolache, "Convergence analysis for a three-step thakur iteration for suzuki-type nonexpansive mappings with visualization," Symmetry, vol. 11, no. 12, p. 1441, 2019.

[23] G. I. Usurelu, A. Bejenaru, and M. Postolache, "Operators with property (E) as concerns numerical analysis and visualization," Numerical Functional Analysis and Optimization, vol. 41, no. 11, pp. 1398-1419, 2020.

[24] M. Bridson and A. Haiger, Metric Spaces of Non-Positive Curvature, Springer-Verlag, Berlin, Germany, 1999.

[25] D. Burago, Y. Burago, and S. Ivanov, "A course in metric geometry," Graduate Studies in Mathematics, Vol. 33, American Mathematical Society, Providence, RI, USA, 2001.

[26] D. Gopal and J. Shilpi, "Fixed point theory in partial metric spaces," in Book Metric Structures and Fixed Point TheoryCRC Press, Boca Raton, FL, USA, 2021.

[27] J. Vishal and J. Shilpi, "G-metric spaces: from the perspective of F-contractions and best proximity points," in Book: Metric Structures and Fixed Point TheoryCRC Press, Boca Raton, FL, USA, 2021.

[28] S. Dhompongsa and B. Panyanak, "On $\Delta$-convergence theorems in CAT (0) spaces," Computers \& Mathematics with Applications, vol. 56, no. 10, pp. 2572-2579, 2008.

[29] S. Dhompongsa, W. A. Kirk, and B. Sims, "Fixed points of uniformly lipschitzian mappings," Nonlinear Analysis: Theory, Methods \& Applications, vol. 65, no. 4, pp. 762-772, 2006.

[30] Z. Opial, "Weak convergence of the sequence of successive approximations for nonexpansive mappings," Bulletin of the American Mathematical Society, vol. 73, no. 4, pp. 591-598, 1967.

[31] W. A. Kirk and B. Panyanak, "A concept of convergence in geodesic spaces," Nonlinear Analysis: Theory, Methods \& Applications, vol. 68, no. 12, pp. 3689-3696, 2008.

[32] S. Dhompongsa, W. A. Kirk, and B. Panyanak, "Nonexpansive set-valued mappings in metric and banach spaces," Journal of Nonlinear and Convex Analysis, vol. 8, pp. 35-45, 2007.

[33] W. Lawaong and B. Panyanak, "Approximating fixed points of nonexpansive nonself mappings in CAT (0) spaces," Fixed Point Theory and Applications, vol. 2010, Article ID 367274, 2009.

[34] H. F. Senter and W. G. Dotson, "Approximating fixed points of nonexpansive mappings," Proceedings of the American Mathematical Society, vol. 44, no. 2, p. 375, 1974.

[35] E. M. Picard, "Memorie sur la theorie des equations aux derivees partielles et lamethode des approximations successives," Journal de Mathématiques Pures et Appliquées, vol. 6, pp. 145-210, 1890.

[36] T. Takahashi, "A convexity in metric spaces and nonexpansive mappings,” Kodai Mathematical Journal, vol. 22, pp. 142-149, 1970.

[37] K. Ullah, J. Ahmad, and M. de la Sen, "Some new results on a three-step iteration process," Axioms, vol. 9, no. 3, p. 110, 2020 . 